\title{
Employment of Artificial Intelligence Techniques for the Optimum Way of Enhanced Oil Recovery
}

Pandian M Vassant ${ }^{1^{*}}$, Junzo Watada ${ }^{1}$, Eswaran Padmanabhan ${ }^{1}$, and Utko Kose ${ }^{2}$

${ }^{1}$ EOR MOR, Universiti Teknologi PETRONAS, 31750 Tronoh, Malaysia

${ }^{2}$ Computer Sciences Application and Research Center, Usak University, Turkey

*Corresponding author: Pandian M Vassant, EOR MOR, Universiti Teknologi PETRONAS, 31750 Tronoh, Malaysia, Tel: 605368 8000; E-mail: pvasant@gmail.com

Received date: Jan 10, 2018; Accepted date: Feb 21, 2018; Published date: Feb 26, 2018

Copyright: (C) 2018 Vassant PM, et al. This is an open-access article distributed under the terms of the Creative Commons Attribution License, which permits unrestricted use, distribution, and reproduction in any medium, provided the original author and source are credited.

Abbreviations EOR: Enhanced Oil Recovery; MOR: Mission Oriented Research

\section{The Issue of Oil Recovery}

For the current age of the 'brave new world', there are many issues associated with especially energy sources of the planet world. Oil is known as one of the most remarkable topic related to the issues on energy sources. Nowadays, we can say that oil is a backbone of every country. Therefore, accounting the inherit uncertainties in respect of survey data which includes the information quality, data resolution and its relative scarcity over the broad spectrum of reservoir characteristics is quite important and the quality of this accounting method directly impacts on the revenue of any country [1]. So, it is important to employ alternative methods for solving the issue of oil recovery in this manner. Although various accounting mythologies are presented in various literatures but all of them either used the statistical method or the Fuzzy Logic, which is an Artificial Intelligence technique $[1,2]$. Because of its effective problem solving approaches, Artificial Intelligence can be used for the issue of oil recovery. In order to understand more about that perspective, it is important to focus on the Artificial Intelligence firstly.

\section{Artificial Intelligence Briefly}

Artificial Intelligence is briefly the intelligence exhibited by machines $[3,4]$. In computer science, an ideal "intelligent" machine is a flexible rational agent that perceives its environment and takes actions that maximize its chance of success at some goal. Colloquially, the term "artificial intelligence" is applied when a machine mimics "cognitive" functions that humans associate with other human minds, such as "learning" and "problem solving" [4,5]. The central problems (or goals) of Artificial Intelligence research include reasoning, knowledge, planning, learning, natural language processing (communication), perception and the ability to move and manipulate objects. Many tools are used in Artificial Intelligence, including versions of search and mathematical optimization, logic, methods based on probability and economics. The Artificial Intelligence field draws upon computer science, mathematics, psychology, linguistics, philosophy, neuroscience and artificial psychology.

\section{Employing Artificial Intelligence for the Issue of Oil Recovery}

There are several reasons that causes oil organizations to do struggle for successful estimation of risk and to find out the optimized method to recovery of oil [1]. For instance, organizations use survey data on which risk estimation and optimal method for the recovery of oil is based $[1,4]$. But the big question arises when these date refer the quality. The wellness of the methodology to provide the matrices to quality is the very beginning of the wellness of any strategy to estimate the risk and to optimize the recovery of oil. When we consider Artificial Intelligence at this point, it is possible to focus on the various survey data to analysis it and develop a framework, which will provide an optimal way for oil recovery. In detail, the quality expect of the data can be exploited to find the optimal way of oil recovery.

When we consider an alternative approach via Artificial Intelligence, it is possible to express details of it as follows: At the initial phase, a Bayesian Analysis of the acquired sample data space is employed. In the next phase, an aggression process is carried thanks to the Bayesian linear regression, Bayesian Estimator or Approximate Bayesian computation. Finally, the last phase includes the mapping the results of the regression to the Fuzzy Logic based system and running an Artificial Intelligence based algorithm to optimize the regression (Figure 1) At this point, the last phase results out a framework.

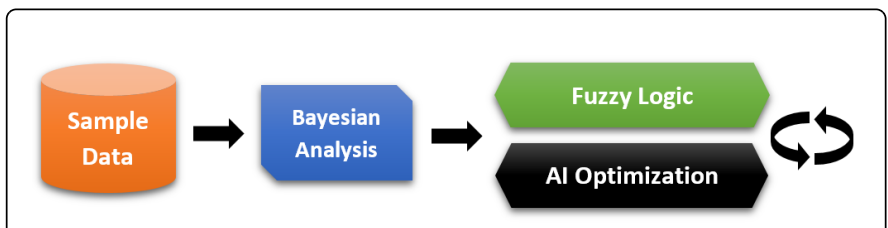

Figure 1: An Artificial Intelligence approach on optimum way of Enhanced Oil Recovery.

The outcome of the expressed Artificial Intelligence approach is associated with boiling up the inherit uncertainties in survey data, which deals with broad spectrum of reservoir characteristics and optimizing the various enhanced oil recovery methods. In this way, advantages of the Artificial Intelligence on real world based problems are proved over the issue of oil recovery, which is an important research point as indicated at early sections of this text.

\section{References}

1. Guo Y, Meng X, Wang D, Meng T, Liu S et al. (2016) Comprehensive risk evaluation of long-distance oil and gas transportation pipelines using a fuzzy Petri net model. J Nat Gas Sci Eng 33: 18-29.

2. Ghiasi MM, Bahadori A, Zendehboudi S (2014) Estimation of triethylene glycol (TEG) purity in natural gas dehydration units using fuzzy neural network. J Nat Gas Sci Eng 17: 26-32.

3. Chen WL, Kan CD, Lin CH, Chen $\mathrm{T}$ (2014) A rule-based decisionmaking diagnosis system to evaluate arteriovenous shunt stenosis for hemodialysis treatment of patients using fuzzy petri nets. IEEE Journal of Biomedical and Health Informatics 18: 703-713. 
Citation: Vassant PM, Watada J, Padmanabhan E, Kose U, et al. (2018) Employment of Artificial Intelligence Techniques for the Optimum Way of Enhanced Oil Recovery. Ind Eng Manage 7: 246. doi:10.4172/2169-0316.1000246

Page 2 of 2

4. Ghiasi MM, Mohammadi AH (2013) Determination of Methane-Hydrate Phase Equilibrium in the Presence of Electrolytes or Organic Inhibitors by using a Semi-Theoretical Framework. Energy Technology 1: 519-529.
5. Hornik K, Stinchcombe M, White H (1989) Multilayer feedforward networks are universal approximators. Neural Networks 2: 359-366. 